\title{
First Measurement of the Form Factors in $D_{s}^{+} \rightarrow K^{0} e^{+} \nu_{e}$ and $D_{s}^{+} \rightarrow K^{* 0} e^{+} \nu_{e}$ Decays
}

M. Ablikim, ${ }^{1}$ M. N. Achasov, ${ }^{10, d}$ S. Ahmed, ${ }^{15}$ M. Albrecht, ${ }^{4}$ M. Alekseev, ${ }^{55 a, 55 c}$ A. Amoroso, ${ }^{55 a, 55 c}$ F. F. An, ${ }^{1}$ Q. An,${ }^{52,42}$ Y. Bai, ${ }^{41}$ O. Bakina, ${ }^{27}$ R. Baldini Ferroli, ${ }^{23 a}$ Y. Ban, ${ }^{35}$ K. Begzsuren, ${ }^{25}$ D. W. Bennett, ${ }^{22}$ J. V. Bennett, ${ }^{5}$ N. Berger, ${ }^{26}$ M. Bertani, ${ }^{23 a}$ D. Bettoni, ${ }^{24 a}$ F. Bianchi,${ }^{55 a, 55 c}$ E. Boger ${ }^{27, b}$ I. Boyko, ${ }^{27}$ R. A. Briere,${ }^{5}$ H. Cai,${ }^{57}$ X. Cai,${ }^{1,42}$ A. Calcaterra, ${ }^{23 a}$ G. F. Cao, ${ }^{1,46}$ S. A. Cetin, ${ }^{45 b}$ J. Chai, ${ }^{55 c}$ J. F. Chang, ${ }^{1,42}$ W. L. Chang, ${ }^{1,46}$ G. Chelkov, ${ }^{27, b, c}$ G. Chen, ${ }^{1}$ H. S. Chen, ${ }^{1,46}$ J. C. Chen, ${ }^{1}$ M. L. Chen, ${ }^{1,42}$ P. L. Chen, ${ }^{53}$ S. J. Chen, ${ }^{33}$ X. R. Chen, ${ }^{30}$ Y. B. Chen, ${ }^{1,42}$ W. Cheng, ${ }^{55}$ X. K. Chu, ${ }^{35}$ G. Cibinetto, ${ }^{24 \mathrm{a}}$ F. Cossio, ${ }^{55 \mathrm{c}}$ H. L. Dai, ${ }^{1,42}$ J. P. Dai, ${ }^{37, \mathrm{~h}}$ A. Dbeyssi, ${ }^{15}$ D. Dedovich ${ }^{27}$ Z. Y. Deng, ${ }^{1}$ A. Denig, ${ }^{26}$ I. Denysenko, ${ }^{27}$ M. Destefanis, ${ }^{55,55 c}$ F. De Mori,${ }^{55 a, 55 c}$ Y. Ding, ${ }^{31}$ C. Dong, ${ }^{34}$ J. Dong, ${ }^{1,42}$ L. Y. Dong, ${ }^{1,46}$ M. Y. Dong, ${ }^{1,42,46}$ Z. L. Dou, ${ }^{33}$ S. X. Du,${ }^{60}$ P. F. Duan, ${ }^{1}$ J. Fang, ${ }^{1,42}$ S. S. Fang, ${ }^{1,46}$ Y. Fang, ${ }^{1}$ R. Farinelli, ${ }^{24 a, 24 b}$ L. Fava, ${ }^{55,55 c}$ S. Fegan, ${ }^{26}$ F. Feldbauer, ${ }^{4}$ G. Felici, ${ }^{23 a}$ C. Q. Feng, ${ }^{52,42}$ E. Fioravanti, ${ }^{24 a}$ M. Fritsch, ${ }^{4}$ C. D. Fu, ${ }^{1}$ Q. Gao, ${ }^{1}$ X. L. Gao,${ }^{52,42}$ Y. Gao, ${ }^{44}$ Y. G. Gao, ${ }^{6}$ Z. Gao, ${ }^{52,42}$ B. Garillon, ${ }^{26}$ I. Garzia, ${ }^{24 a}$ A. Gilman, ${ }^{49}$ K. Goetzen, ${ }^{11}$ L. Gong, ${ }^{34}$ W. X. Gong, ${ }^{1,42}$ W. Gradl, ${ }^{26}$ M. Greco, ${ }^{55 a, 55 \mathrm{c}}$ L. M. Gu,${ }^{33}$ M. H. Gu,${ }^{1,42}$ Y. T. Gu, ${ }^{13}$ A. Q. Guo, ${ }^{1}$ L. B. Guo, ${ }^{32}$ R. P. Guo, ${ }^{1,46}$ Y. P. Guo, ${ }^{26}$ A. Guskov ${ }^{27}$ Z. Haddadi, ${ }^{29}$ S. Han,${ }^{57}$ X. Q. Hao,${ }^{16}$ F. A. Harris, ${ }^{47}$ K. L. He, ${ }^{1,46}$ X. Q. He,${ }^{51}$ F. H. Heinsius, ${ }^{4}$ T. Held, ${ }^{4}$ Y. K. Heng, ${ }^{1,42,46}$ Z. L. Hou, ${ }^{1}$ H. M. Hu, ${ }^{1,46}$ J. F. Hu, ${ }^{37, h}$ T. Hu, ${ }^{1,42,46}$ Y. Hu, ${ }^{1}$ G. S. Huang, ${ }^{52,42}$ J. S. Huang, ${ }^{16}$ X. T. Huang, ${ }^{36}$ X. Z. Huang, ${ }^{33}$

Z. L. Huang, ${ }^{31}$ T. Hussain, ${ }^{54}$ W. Ikegami Andersson, ${ }^{56}$ M. Irshad,${ }^{52,42}$ Q. Ji, ${ }^{1}$ Q. P. Ji ${ }^{16}$ X. B. Ji, ${ }^{1,46}$ X. L. Ji, ${ }^{1,42}$ X. S. Jiang,,${ }^{1,42,46}$ X. Y. Jiang, ${ }^{34}$ J. B. Jiao, ${ }^{36}$ Z. Jiao, ${ }^{18}$ D. P. Jin, ${ }^{1,42,46}$ S. Jin, ${ }^{33}$ Y. Jin, ${ }^{48}$ T. Johansson, ${ }^{56}$ A. Julin, ${ }^{49}$ N. Kalantar-Nayestanaki, ${ }^{29}$ X. S. Kang, ${ }^{34}$ M. Kavatsyuk, ${ }^{29}$ B. C. Ke, ${ }^{1}$ I. K. Keshk, ${ }^{4}$ T. Khan,${ }^{52,42}$ A. Khoukaz, ${ }^{50}$ P. Kiese, ${ }^{26}$ R. Kiuchi, ${ }^{1}$ R. Kliemt, ${ }^{11}$ L. Koch ${ }^{28}$ O. B. Kolcu, ${ }^{45 b, \mathrm{f}}$ B. Kopf ${ }^{4}$ M. Kornicer, ${ }^{47}$ M. Kuemmel, ${ }^{4}$ M. Kuessner, ${ }^{4}$ A. Kupsc, ${ }^{56}$ M. Kurth, ${ }^{1}$ W. Kühn, ${ }^{28}$ J. S. Lange, ${ }^{28}$ P. Larin, ${ }^{15}$ L. Lavezzi, ${ }^{55 c}$ S. Leiber, ${ }^{4}$ H. Leithoff, ${ }^{26}$ C. Li, ${ }^{56}$ Cheng Li, ${ }^{52,42}$ D. M. Li ${ }^{60}$ F. Li, ${ }^{1,42}$ F. Y. Li ${ }^{35}$ G. Li ${ }^{1}{ }^{1}$ H. B. Li, ${ }^{1,46}$ H. J. Li, ${ }^{1,46}$ J. C. Li ${ }^{1}$ J. W. Li ${ }^{40}$ K. J. Li, ${ }^{43}{ }^{K a n g ~ L i},{ }^{14} \mathrm{Ke} \mathrm{Li},{ }^{1} \mathrm{Lei} \mathrm{Li},{ }^{3}{ }^{*}$ P. L. Li, ${ }^{52,42}$ P. R. Li, ${ }^{46,7}$ Q. Y. Li ${ }^{36}$ T. Li, ${ }^{36}$ W. D. Li, ${ }^{1,46}$ W. G. Li, ${ }^{1}$ X. L. Li, ${ }^{36}$ X. N. Li, ${ }^{1,42}$ X. Q. Li, ${ }^{34}$ Z. B. Li, ${ }^{43}$ H. Liang, ${ }^{52,42}$ Y. F. Liang, ${ }^{39}$ Y. T. Liang ${ }^{28}$ G. R. Liao, ${ }^{12}$ L. Z. Liao, ${ }^{1,46}$ J. Libby, ${ }^{21}$ C. X. Lin,${ }^{43}$ D. X. Lin,${ }^{15}$ B. Liu, ${ }^{37, h}$ B. J. Liu, ${ }^{1}$ C. X. Liu, ${ }^{1}$ D. Liu, ${ }^{52,42}$ D. Y. Liu, ${ }^{37, h}$ F. H. Liu ${ }^{38}$ Fang Liu, ${ }^{1}$ Feng Liu, ${ }^{6}$ H. B. Liu ${ }^{13}$ H. L. Liu, ${ }^{41}$ H. M. Liu, ${ }^{1,46}$ Huanhuan Liu, ${ }^{1}$ Huihui Liu, ${ }^{17}$ J. B. Liu, ${ }^{52,42}$ J. Y. Liu, ${ }^{1,46}$ K. Y. Liu, ${ }^{31}$ Ke Liu, ${ }^{6}$ L. D. Liu, ${ }^{35}$ Q. Liu, ${ }^{46}$ S. B. Liu, ${ }^{52,42}$ X. Liu, ${ }^{30}$ Y. B. Liu ${ }^{34}$ Z. A. Liu, ${ }^{1,42,46}$ Zhiqing Liu, ${ }^{26}$ Y. F. Long, ${ }^{35}$ X. C. Lou, ${ }^{1,42,46}$ H. J. Lu, ${ }^{18}$ J. G. Lu, ${ }^{1,42}$ Y. Lu, ${ }^{1}$ Y. P. Lu, ${ }^{1,42}$ C. L. Luo, ${ }^{32}$ M. X. Luo, ${ }^{59}$ T. Luo, ${ }^{9, j}$ X. L. Luo, ${ }^{1,42}$ S. Lusso, ${ }^{55 c}$ X. R. Lyu, ${ }^{46}$ F. C. Ma, ${ }^{31}$ H. L. Ma, ${ }^{1}$ L. L. Ma, ${ }^{36}$ M. M. Ma, ${ }^{1,46}$ Q. M. Ma, ${ }^{1}$ X. N. Ma ${ }^{34}$ X. Y. Ma, ${ }^{1,42}$ Y. M. Ma, ${ }^{36}$ F. E. Maas, ${ }^{15}$ M. Maggiora, ${ }^{55,55 c}$ S. Maldaner, ${ }^{26}$ Q. A. Malik, ${ }^{54}$ A. Mangoni, ${ }^{23 b}$ Y. J. Mao, ${ }^{35}$ Z. P. Mao, ${ }^{1}$ S. Marcello, ${ }^{55 a, 55 c}$ Z. X. Meng, ${ }^{48}$ J. G. Messchendorp, ${ }^{29}$ G. Mezzadri, ${ }^{24 a}$ J. Min, ${ }^{1,42}$ T. J. Min, ${ }^{33}$ R. E. Mitchell, ${ }^{22}$ X. H. Mo, ${ }^{1,42,46}$ Y. J. Mo, ${ }^{6}$ C. Morales Morales, ${ }^{15}$ N. Yu. Muchnoi, ${ }^{10, d}$ H. Muramatsu, ${ }^{49}$ A. Mustafa, ${ }^{4}$

S. Nakhoul, ${ }^{11, g}$ Y. Nefedov, ${ }^{27}$ F. Nerling, ${ }^{11, g}$ I. B. Nikolaev, ${ }^{10, d}$ Z. Ning, ${ }^{1,42}$ S. Nisar, ${ }^{8}$ S. L. Niu, ${ }^{1,42}$ X. Y. Niu, ${ }^{1,46}$ S. L. Olsen, ${ }^{46}$ Q. Ouyang, ${ }^{1,42,46}$ S. Pacetti, ${ }^{23 b}$ Y. Pan, ${ }^{52,42}$ M. Papenbrock, ${ }^{56}$ P. Patteri, ${ }^{23 a}$ M. Pelizaeus, ${ }^{4}$ J. Pellegrino, ${ }^{55 a, 55 c}$ H. P. Peng, ${ }^{52,42}$ Z. Y. Peng, ${ }^{13}$ K. Peters,${ }^{11,9}$ J. Pettersson, ${ }^{56}$ J. L. Ping, ${ }^{32}$ R. G. Ping, ${ }^{1,46}$ A. Pitka, ${ }^{4}$ R. Poling, ${ }^{49}$ V. Prasad,${ }^{52,42}$ H. R. Qi, ${ }^{2}$ M. Qi ${ }^{33}$ T. Y. Qi ${ }^{2}$ S. Qian, ${ }^{1,42}$ C. F. Qiao, ${ }^{46}$ N. Qin, ${ }^{57}$ X. S. Qin, ${ }^{4}$ Z.H. Qin, ${ }^{1,42}$ J. F. Qiu, ${ }^{1}$ S. Q. Qu, ${ }^{34}$

K. H. Rashid, ${ }^{54, \mathrm{i}}$ C. F. Redmer, ${ }^{26}$ M. Richter, ${ }^{4}$ M. Ripka, ${ }^{26}$ A. Rivetti,${ }^{55 \mathrm{c}}$ M. Rolo, ${ }^{55 \mathrm{c}}$ G. Rong, ${ }^{1,46}$ Ch. Rosner, ${ }^{15}$ A. Sarantsev, ${ }^{27, e}$ M. Savrié, ${ }^{24 b}$ K. Schoenning, ${ }^{56}$ W. Shan, ${ }^{19}$ X. Y. Shan,${ }^{52,42}$ M. Shao, ${ }^{52,42}$ C. P. Shen, ${ }^{2}$ P. X. Shen, ${ }^{34}$ X. Y. Shen,${ }^{1,46}$ H. Y. Sheng, ${ }^{1}$ X. Shi, ${ }^{1,42}$ J. J. Song, ${ }^{36}$ W. M. Song, ${ }^{36}$ X. Y. Song, ${ }^{1}$ S. Sosio, ${ }^{55 a, 55 c}$ C. Sowa, ${ }^{4}$ S. Spataro, ${ }^{55 a, 55 c}$ G. X. Sun, ${ }^{1}$ J. F. Sun, ${ }^{16}$ L. Sun, ${ }^{57}$ S. S. Sun, ${ }^{1,46}$ X. H. Sun, ${ }^{1}$ Y. J. Sun, ${ }^{52,42}$ Y. K. Sun, ${ }^{52,42}$ Y. Z. Sun, ${ }^{1}$ Z. J. Sun, ${ }^{1,42}$ Z. T. Sun, ${ }^{1}$ Y. T. Tan, ${ }^{52,42}$ C. J. Tang, ${ }^{39}$ G. Y. Tang, ${ }^{1}$ X. Tang, ${ }^{1}$ M. Tiemens, ${ }^{29}$ B. Tsednee, ${ }^{25}$ I. Uman, ${ }^{45 \mathrm{~d}}$ B. Wang, ${ }^{1}$ B. L. Wang, ${ }^{46}$ C. W. Wang, ${ }^{33}$ D. Wang, ${ }^{35}$ D. Y. Wang, ${ }^{35}$ Dan Wang, ${ }^{46}$ K. Wang, ${ }^{1,42}$ L. L. Wang, ${ }^{1}$ L. S. Wang, ${ }^{1}$ M. Wang, ${ }^{36}$ Meng Wang,, 46 P. Wang, ${ }^{1}$ P. L. Wang, ${ }^{1}$ W. P. Wang, ${ }^{52,42}$ X. F. Wang, ${ }^{1}$ Y. Wang, ${ }^{52,42}$ Y. F. Wang, ${ }^{1,42,46}$ Z. Wang, ${ }^{1,42}$ Z. G. Wang, ${ }^{1,42}$ Z. Y. Wang, ${ }^{1}$ Zongyuan Wang, ${ }^{1,46}$ T. Weber, ${ }^{4}$ D. H. Wei, ${ }^{12}$ P. Weidenkaff, ${ }^{26}$ S. P. Wen, ${ }^{1}$ U. Wiedner, ${ }^{4}$ M. Wolke, ${ }^{56}$ L. H. Wu, ${ }^{1}$ L. J. Wu, ${ }^{1,46}$ Z. Wu, ${ }^{1,42}$ L. Xia, ${ }^{52,42}$ X. Xia ${ }^{36}$ Y. Xia, ${ }^{20}$ D. Xiao, ${ }^{1}$ Y. J. Xiao, ${ }^{1,46}$ Z. J. Xiao, ${ }^{32}$ Y. G. Xie, ${ }^{1,42}$ Y. H. Xie, ${ }^{6}$ X. A. Xiong, ${ }^{1,46}$ Q. L. Xiu, ${ }^{1,42}$ G. F. Xu, ${ }^{1}$ J. J. Xu, ${ }^{1,46}$ L. Xu, ${ }^{1}$ Q. J. Xu, ${ }^{14}$ X. P. Xu, ${ }^{40}$ F. Yan, ${ }^{53}$ L. Yan, ${ }^{55 a, 55 c}$ W. B. Yan, ${ }^{52,42}$ W. C. Yan, ${ }^{2}$ Y. H. Yan, ${ }^{20}$ H. J. Yang ${ }^{37, h}$ H. X. Yang, ${ }^{1}$ L. Yang,${ }^{57}$ R. X. Yang, ${ }^{52,42}$ S. L. Yang, ${ }^{1,46}$ Y. H. Yang,${ }^{33}$ Y.X. Yang, ${ }^{12}$ Yifan Yang, ${ }^{1,46}$ Z. Q. Yang, ${ }^{20}$ M. Ye, ${ }^{1,42}$ M. H. Ye, ${ }^{7}$ J. H. Yin, ${ }^{1}$ Z. Y. You, ${ }^{43}$ B. X. Yu,,${ }^{1,42,46}$ C. X. Yu, ${ }^{34}$ J. S. Yu, ${ }^{20}$ J. S. Yu, ${ }^{30}$ C. Z. Yuan, ${ }^{1,46}$ Y. Yuan, ${ }^{1}$ A. Yuncu ${ }^{45 b, a}$ A. A. Zafar, ${ }^{54}$ Y. Zeng, ${ }_{58}$ B. X. Zhang, ${ }^{1}$ B. Y. Zhang, ${ }_{1,42}^{1,46}$ C. C. Zhang, ${ }^{1}$ D. H. Zhang, ${ }^{1}$ H. H. Zhang, ${ }^{43}$ H. Y. Zhang, ${ }^{1,42}$ J. Zhang, ${ }^{1,46}$ J. L. Zhang, ${ }^{58}$ J. Q. Zhang, ${ }^{4}$ J. W. Zhang, ${ }^{1,42,46}$ J. Y. Zhang, ${ }_{1}^{1}$ J. Z. Zhang, ${ }^{1,46}$ K. Zhang, ${ }^{1,46}$ L. Zhang, ${ }^{44}$ S. F. Zhang, ${ }^{33}$ T. J. Zhang, ${ }^{37, h}$ X. Y. Zhang, ${ }^{36}$ Y. Zhang, ${ }^{52,42}$ Y. H. Zhang, ${ }^{1,42}$ Y. T. Zhang, ${ }^{52,42}$ Yang Zhang, ${ }^{1}$ Yao Zhang, ${ }^{1}$ Yu Zhang, ${ }^{46}$ Z. H. Zhang, ${ }^{6}$ Z. P. Zhang, ${ }^{52}$ Z. Y. Zhang, ${ }^{57}$ 
G. Zhao, ${ }_{1}^{1}$ J. W. Zhao, ${ }^{1,42}$ J. Y. Zhao, ${ }^{1,46}$ J. Z. Zhao, ${ }^{1,42}$ Lei Zhao, ${ }^{52,42}$ Ling Zhao, ${ }^{1}$ M. G. Zhao, ${ }^{34}$ Q. Zhao, ${ }^{1}$ S. J. Zhao, ${ }^{60}$ T. C. Zhao, ${ }^{1}$ Y. B. Zhao, ${ }^{1,42}$ Z. G. Zhao, ${ }^{52,42}$ A. Zhemchugov, ${ }^{27, b}$ B. Zheng, ${ }^{53}$ J. P. Zheng, ${ }^{1,42}$ W. J. Zheng, ${ }^{36}$ Y. H. Zheng, ${ }^{46}$ B. Zhong, ${ }^{32}$ L. Zhou, ${ }^{1,42}$ Q. Zhou, ${ }^{1,46}$ X. Zhou, ${ }^{57}$ X. K. Zhou, ${ }^{52,42}$ X. R. Zhou, ${ }^{52,42}$ X. Y. Zhou, ${ }^{1}$ Xiaoyu Zhou, ${ }^{20}$ Xu Zhou, ${ }^{20}$ A. N. Zhu, ${ }^{1,46}$ J. Zhu, ${ }^{34}$ J. Zhu, ${ }^{43}$ K. Zhu, ${ }^{1}$ K. J. Zhu, ${ }^{1,42,46}$ S. Zhu, ${ }^{1}$ S. H. Zhu, ${ }^{51}$ X. L. Zhu, ${ }^{44}$ Y. C. Zhu, ${ }^{52,42}$ Y. S. Zhu, ${ }^{1,46}$ Z. A. Zhu, ${ }^{1,46}$ J. Zhuang, ${ }^{1,42}$ B.S. Zou, ${ }^{1}$ and J. H. Zou ${ }^{1}$

\title{
(BESIII Collaboration)
}

\author{
${ }^{1}$ Institute of High Energy Physics, Beijing 100049, People's Republic of China \\ ${ }^{2}$ Beihang University, Beijing 100191, People's Republic of China \\ ${ }^{3}$ Beijing Institute of Petrochemical Technology, Beijing 102617, People's Republic of China \\ ${ }^{4}$ Bochum Ruhr-University, D-44780 Bochum, Germany \\ ${ }^{5}$ Carnegie Mellon University, Pittsburgh, Pennsylvania 15213, USA \\ ${ }^{6}$ Central China Normal University, Wuhan 430079, People's Republic of China \\ ${ }^{7}$ China Center of Advanced Science and Technology, Beijing 100190, People's Republic of China \\ ${ }^{8}$ COMSATS Institute of Information Technology, Lahore, Defence Road, Off Raiwind Road, 54000 Lahore, Pakistan \\ ${ }^{9}$ Fudan University, Shanghai 200443, People's Republic of China \\ ${ }^{10}$ G.I. Budker Institute of Nuclear Physics SB RAS (BINP), Novosibirsk 630090, Russia \\ ${ }^{11}$ GSI Helmholtzcentre for Heavy Ion Research GmbH, D-64291 Darmstadt, Germany \\ ${ }^{12}$ Guangxi Normal University, Guilin 541004, People's Republic of China \\ ${ }^{13}$ Guangxi University, Nanning 530004, People's Republic of China \\ ${ }^{14}$ Hangzhou Normal University, Hangzhou 310036, People's Republic of China \\ ${ }^{15}$ Helmholtz Institute Mainz, Johann-Joachim-Becher-Weg 45, D-55099 Mainz, Germany \\ ${ }^{16}$ Henan Normal University, Xinxiang 453007, People's Republic of China \\ ${ }^{17}$ Henan University of Science and Technology, Luoyang 471003, People's Republic of China \\ ${ }^{18}$ Huangshan College, Huangshan 245000, People's Republic of China \\ ${ }^{19}$ Hunan Normal University, Changsha 410081, People's Republic of China \\ ${ }^{20}$ Hunan University, Changsha 410082, People's Republic of China \\ ${ }^{21}$ Indian Institute of Technology Madras, Chennai 600036, India \\ ${ }^{22}$ Indiana University, Bloomington, Indiana 47405, USA \\ ${ }^{23 a}$ INFN Laboratori Nazionali di Frascati, I-00044, Frascati, Italy \\ ${ }^{23 \mathrm{~b}}$ INFN and University of Perugia, I-06100, Perugia, Italy \\ ${ }^{24 a}$ INFN Sezione di Ferrara, I-44122, Ferrara, Italy \\ ${ }^{24 b}$ University of Ferrara, I-44122, Ferrara, Italy \\ ${ }^{25}$ Institute of Physics and Technology, Peace Avenue 54B, Ulaanbaatar 13330, Mongolia \\ ${ }^{26}$ Johannes Gutenberg University of Mainz, Johann-Joachim-Becher-Weg 45, D-55099 Mainz, Germany \\ ${ }^{27}$ Joint Institute for Nuclear Research, 141980 Dubna, Moscow region, Russia \\ ${ }^{28}$ Justus-Liebig-Universitaet Giessen, II. Physikalisches Institut, Heinrich-Buff-Ring 16, D-35392 Giessen, Germany \\ ${ }^{29}$ KVI-CART, University of Groningen, NL-9747 AA Groningen, Netherlands \\ ${ }^{30}$ Lanzhou University, Lanzhou 730000, People's Republic of China \\ ${ }^{31}$ Liaoning University, Shenyang 110036, People's Republic of China \\ ${ }^{32}$ Nanjing Normal University, Nanjing 210023, People's Republic of China \\ ${ }^{33}$ Nanjing University, Nanjing 210093, People's Republic of China \\ ${ }^{34}$ Nankai University, Tianjin 300071, People's Republic of China \\ ${ }^{35}$ Peking University, Beijing 100871, People's Republic of China \\ ${ }^{36}$ Shandong University, Jinan 250100, People's Republic of China \\ ${ }^{37}$ Shanghai Jiao Tong University, Shanghai 200240, People's Republic of China \\ ${ }^{38}$ Shanxi University, Taiyuan 030006, People's Republic of China \\ ${ }^{39}$ Sichuan University, Chengdu 610064, People's Republic of China \\ ${ }^{40}$ Soochow University, Suzhou 215006, People's Republic of China \\ ${ }^{41}$ Southeast University, Nanjing 211100, People's Republic of China \\ ${ }^{42}$ State Key Laboratory of Particle Detection and Electronics, Beijing 100049, Hefei 230026, People's Republic of China \\ ${ }^{43}$ Sun Yat-Sen University, Guangzhou 510275, People's Republic of China \\ ${ }^{44}$ Tsinghua University, Beijing 100084, People's Republic of China \\ ${ }^{45 a}$ Ankara University, 06100 Tandogan, Ankara, Turkey \\ ${ }^{45 b}$ Istanbul Bilgi University, 34060 Eyup, Istanbul, Turkey \\ ${ }^{45 c}$ Uludag University, 16059 Bursa, Turkey \\ ${ }^{45 \mathrm{~d}}$ Near East University, Nicosia, North Cyprus, Mersin 10, Turkey \\ ${ }^{46}$ University of Chinese Academy of Sciences, Beijing 100049, People's Republic of China
}




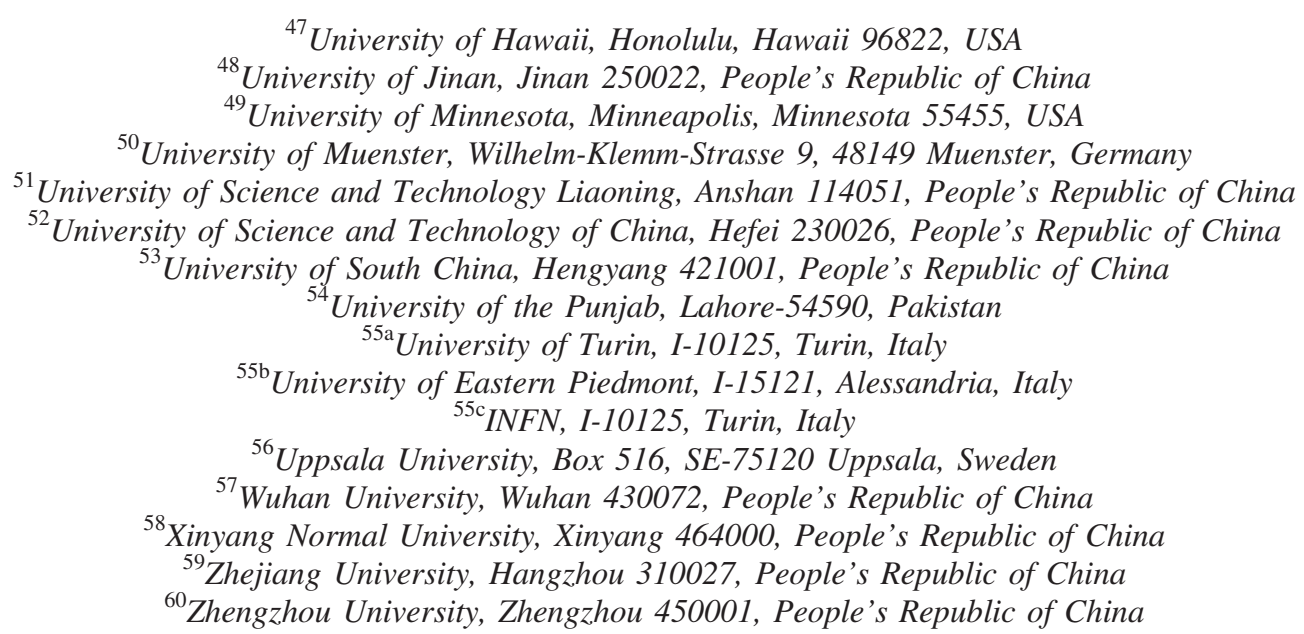

(Received 8 November 2018; published 15 February 2019)

\begin{abstract}
We report on new measurements of Cabibbo-suppressed semileptonic $D_{s}^{+}$decays using $3.19 \mathrm{fb}^{-1}$ of $e^{+} e^{-}$annihilation data sample collected at a center-of-mass energy of $4.178 \mathrm{GeV}$ with the BESIII detector at the BEPCII collider. Our results include branching fractions $\mathcal{B}\left(D_{s}^{+} \rightarrow K^{0} e^{+} \nu_{e}\right)=[3.25 \pm 0.38$ (stat) \pm 0.16 (syst) $] \times 10^{-3}$ and $\mathcal{B}\left(D_{s}^{+} \rightarrow K^{* 0} e^{+} \nu_{e}\right)=[2.37 \pm 0.26$ (stat) \pm 0.20 (syst) $] \times 10^{-3}$, which are much improved relative to previous measurements, and the first measurements of the hadronic form-factor parameters for these decays. For $D_{s}^{+} \rightarrow K^{0} e^{+} \nu_{e}$, we obtain $f_{+}(0)=0.720 \pm 0.084$ (stat) \pm 0.013 (syst), and for $D_{s}^{+} \rightarrow K^{* 0} e^{+} \nu_{e}$, we find form-factor ratios $r_{V}=V(0) / A_{1}(0)=1.67 \pm 0.34$ (stat) \pm 0.16 (syst) and $r_{2}=A_{2}(0) / A_{1}(0)=0.77 \pm 0.28$ (stat) \pm 0.07 (syst).
\end{abstract}

DOI: 10.1103/PhysRevLett.122.061801

The study of $D_{s}^{+}$semileptonic (SL) decays provides valuable information about weak and strong interactions in mesons composed of heavy quarks. (Throughout this Letter, charge-conjugate modes are implied unless explicitly noted.) Measurement of the total SL decay width of the $D_{s}^{+}$, and comparison with that of the $D$ mesons, can help elucidate the role of nonperturbative effects in heavymeson decays [1,2]. The Cabibbo-suppressed (CS) SL decays, including the branching fractions (BFs) for $D_{s}^{+} \rightarrow$ $K^{0} e^{+} \nu_{e}$ and $D_{s}^{+} \rightarrow K^{* 0} e^{+} \nu_{e}$ [3], are especially poorly measured. Detailed investigations of the dynamics of these decays allow measurements of SL decay partial widths, which depend on the hadronic form factors (FFs) describing the interaction between the final-state quarks. Measurements of these FFs provide experimental tests of theoretical predictions of lattice QCD (LQCD). Reference [4] predicts that the FFs have minimal dependence on the spectator-quark mass, with values for $D_{s}^{+} \rightarrow$ $K^{0} \ell^{+} \nu_{\ell}$ and $D^{+} \rightarrow \pi^{0} \ell^{+} \nu_{\ell}$ differing by less than $5 \%$. Experimental verification of this predicted instance would

Published by the American Physical Society under the terms of the Creative Commons Attribution 4.0 International license. Further distribution of this work must maintain attribution to the author(s) and the published article's title, journal citation, and DOI. Funded by SCOAP . be a significant success for LQCD. A complementary LQCD test is provided by comparing measured and predicted FF parameters for $D_{s}^{+} \rightarrow K^{* 0} \ell^{+} \nu_{\ell}$ and $D^{+} \rightarrow$ $\rho^{0} \ell^{+} \nu_{\ell}$. The combination of these measurements has the potential to verify LQCD FF predictions for SL charm decays to both pseudoscalar and vector mesons, useful for further applying the LQCD to SL $B$ decays for precise determination of Cabibbo-Kobayashi-Maskawa (CKM) parameters [4-6].

In this Letter, we report on improved measurements of the absolute BFs and first measurements of the FFs for the decays $D_{s}^{+} \rightarrow K^{0} e^{+} \nu_{e}$ and $D_{s}^{+} \rightarrow K^{* 0} e^{+} \nu_{e}$. Our measurements have been made with $3.19 \mathrm{fb}^{-1} e^{+} e^{-}$annihilation data recorded with the BESIII detector at the BEPCII collider. The center-of-mass energy for our data is $\sqrt{s}=4.178 \mathrm{GeV}$. The cross section is $\sim 1 \mathrm{nb}$ for the production of $D_{s}^{*+} D_{s}^{-}+$c.c. at this energy. Our data sample is the largest collected by any experiment for $D_{s}^{+}$studies in the clean near-threshold environment.

Details about the BESIII detector design and performance are provided in Ref. [7]. A GEANT4-based [8] Monte Carlo (MC) simulation package, which includes the geometric description of the detector and the detector response, is used to determine signal detection efficiencies and to estimate potential backgrounds. Signal MC samples of $e^{+} e^{-} \rightarrow D_{s}^{*+} D_{s}^{-}$with a $D_{s}^{+}$meson decaying to 
$K^{(*) 0} e^{+} \nu_{e}$ together with a $D_{s}^{-}$decaying to the studied decay modes used for this analysis are generated with CONEXC [9] using EVTGEN [10], with the inclusion of initial-state radiation (ISR) effects up to second-order correction $[9,11]$. The final-state radiation (FSR) effects are simulated via the PHOTOS package [12]. The interference effects between ISR and FSR are ignored [13]. The simulation of the SL decay $D_{s}^{+} \rightarrow K^{*(0)} e^{+} \nu_{e}$ is matched with the FFs measured in this work. To study the backgrounds, inclusive MC samples consisting of open-charm states, radiative return to $J / \psi$ and $\psi(2 S)$, and continuum processes of $q \bar{q}$ $(q=u, d, s)$, along with Bhabha scattering $\mu^{+} \mu^{-}, \tau^{+} \tau^{-}$, and $\gamma \gamma$ events are generated. All known decay modes of open-charm and $\psi$ states are simulated as specified by the Particle Data Group [14], while the remaining unknown decays are modeled with LUNDCHARM [15].

As described above, $D_{s}^{+}$mesons are produced at $\sqrt{s}=$ $4.178 \mathrm{GeV}$ predominantly through $D_{s}^{*+} D_{s}^{-}$[16], with $94 \%$ of the $D_{s}^{*+}$ decaying to $\gamma D_{s}^{+}$. The first step of our analysis is to select "single-tag" (ST) events with a fully reconstructed $D_{s}^{-}$candidate. The $D_{s}^{-}$hadronic decay tag modes that are used for this analysis are listed in Table I. In this ST sample, we select the SL decay $D_{s}^{+} \rightarrow K^{(*) 0} e^{+} \nu_{e}$ plus an isolated photon consistent with being from the $D_{s}^{*} \rightarrow \gamma D_{s}$ transition. The selected events are referred to as the double-tag (DT) sample. For a specific tag mode $i$, the ST and DT event yields can be expressed as

$$
N_{\mathrm{ST}}^{i}=2 N_{D_{s} D_{s}^{*}} \mathcal{B}_{\mathrm{ST}}^{i} \epsilon_{\mathrm{ST}}^{i} \quad \text { and } \quad N_{\mathrm{DT}}^{i}=2 N_{D_{s} D_{s}^{*}} \mathcal{B}_{\mathrm{ST}}^{i} \mathcal{B}_{\mathrm{SL}}^{i} \epsilon_{\mathrm{DT}}^{i},
$$

where $N_{D_{s} D_{s}^{*}}$ is the number of $D_{s} D_{s}^{*}$ pairs, $\mathcal{B}_{\mathrm{ST}}^{i}$ and $\mathcal{B}_{\mathrm{SL}}^{i}$ are the BFs of the $D_{s}^{-}$tag mode and the $D_{s}^{+}$SL decay mode, respectively, $\epsilon_{\mathrm{ST}}^{i}$ is the efficiency for finding the tag candidate, and $\epsilon_{\mathrm{DT}}^{i}$ is the efficiency for simultaneously finding the tag $D_{s}^{-}$and the SL decay. The DT efficiency $\epsilon_{\mathrm{DT}}^{i}$

TABLE I. $M_{D_{s}^{-}}$windows and ST yields in data.

\begin{tabular}{lcr}
\hline \hline ST mode & $M_{D_{s}^{-}}\left(\mathrm{GeV} / c^{2}\right)$ & \multicolumn{1}{c}{$N_{\mathrm{ST}}^{i}$} \\
\hline$K_{S}^{0} K^{-}$ & $(1.945,1.990)$ & $25858 \pm 217$ \\
$K^{+} K^{-} \pi^{-}$ & $(1.945,1.990)$ & $130666 \pm 575$ \\
$K_{S}^{0} K^{-} \pi^{0}$ & $(1.940,1.990)$ & $10807 \pm 398$ \\
$K_{S}^{0} K_{S}^{0} \pi^{-}$ & $(1.945,1.990)$ & $3810 \pm 131$ \\
$K^{+} K^{-} \pi^{-} \pi^{0}$ & $(1.940,1.990)$ & $35091 \pm 702$ \\
$K_{S}^{0} K^{-} \pi^{+} \pi^{-}$ & $(1.945,1.990)$ & $7722 \pm 235$ \\
$K_{S}^{0} K^{+} \pi^{-} \pi^{-}$ & $(1.945,1.990)$ & $14802 \pm 259$ \\
$\pi^{+} \pi^{-} \pi^{-}$ & $(1.945,1.990)$ & $36258 \pm 832$ \\
$\pi^{-} \eta$ & $(1.940,1.990)$ & $17535 \pm 400$ \\
$\rho^{-} \eta$ & $(1.940,1.990)$ & $30114 \pm 886$ \\
$\pi^{-} \eta^{\prime}\left(\eta^{\prime} \rightarrow \pi^{+} \pi^{-} \eta\right)$ & $(1.940,1.990)$ & $7704 \pm 152$ \\
$\rho^{-} \eta^{\prime}\left(\eta^{\prime} \rightarrow \pi^{+} \pi^{-} \eta\right)$ & $(1.940,1.990)$ & $3039 \pm 226$ \\
$\pi^{-} \eta^{\prime}\left(\eta^{\prime} \rightarrow \gamma \pi^{+} \pi^{-}\right)$ & $(1.940,1.990)$ & $17919 \pm 481$ \\
\hline \hline
\end{tabular}

includes the $\mathrm{BF}$ for $D_{s}^{*+} \rightarrow \gamma D_{s}^{+}$. The BF for the SL decay is given by

$$
\mathcal{B}_{\mathrm{SL}}=\frac{N_{\mathrm{DT}}}{\sum N_{\mathrm{ST}}^{i} \times \epsilon_{\mathrm{DT}}^{i} / \epsilon_{\mathrm{ST}}^{i}}=\frac{N_{\mathrm{DT}}}{N_{\mathrm{ST}} \times \epsilon_{\mathrm{SL}}},
$$

where $N_{\mathrm{DT}}$ is the total yield of DT events, $N_{\mathrm{ST}}$ is the total ST yield, and $\epsilon_{\mathrm{SL}}=\left[\left(\sum N_{\mathrm{ST}}^{i} \times \epsilon_{\mathrm{DT}}^{i} / \epsilon_{\mathrm{ST}}^{i}\right) /\left(\sum N_{\mathrm{ST}}^{i}\right)\right]$ is the average efficiency for finding the SL decay weighted by the measured yields of the tag modes in data.

Selection criteria for $\gamma, \pi^{ \pm}$, and $K^{ \pm}$are the same as those used in Ref. [17]. The $\pi^{0}(\eta)$ candidate is reconstructed from the $\gamma \gamma$ combination with invariant mass within $(0.115,0.150)[(0.50,0.57)] \mathrm{GeV} / c^{2}$. To improve the momentum resolution, a kinematic fit is performed to constrain $\gamma \gamma$ invariant mass to the nominal $\pi^{0}(\eta)$ mass [3] with $\chi^{2}<20$. The fitted $\pi^{0}(\eta)$ momenta are used for further analysis. $K_{S}^{0}$ mesons are reconstructed from two oppositely charged tracks with its invariant mass within $(0.485,0.510) \mathrm{GeV} / c^{2}$. A vertex constraint is applied to improve the $K_{S}^{0}$ signal significance as in Ref. [18]. We select $\rho^{-} \rightarrow \pi^{-} \pi^{0}$ by requiring the invariant mass $M_{\pi^{-} \pi^{0}}$ to be within $(0.626,0.924) \mathrm{GeV} / c^{2}$ [3]. The decay modes $\eta^{\prime} \rightarrow \pi^{+} \pi^{-} \eta$ and $\eta^{\prime} \rightarrow \gamma \pi^{+} \pi^{-}$are used to select $\eta^{\prime}$ mesons, with the invariant masses of the $\pi^{+} \pi^{-} \eta$ and $\gamma \pi^{+} \pi^{-}$required to be within $(0.940,0.976)$ and $(0.940,0.970) \mathrm{GeV} / c^{2}$, respectively. Additionally, to suppress backgrounds from $D^{*}$ decays, the momenta of the photons from $\eta^{\prime} \rightarrow \gamma \pi^{+} \pi^{-}$ and all pions are required to be greater than $0.1 \mathrm{GeV} / c$.

For all events passing the ST selection criteria, we calculate the recoil mass against the tag with the following formula:

$$
M_{\mathrm{rec}}=\sqrt{\left(\sqrt{s}-\sqrt{\left|\vec{p}_{D_{s}^{-}}\right|^{2}+m_{D_{s}^{-}}^{2}}\right)^{2}-\left|\vec{p}_{D_{s}^{-}}\right|^{2}},
$$

where $m_{D_{s}^{-}}$and $\vec{p}_{D_{s}^{-}}$are the known mass [3] and measured momentum of the tag $D_{s}^{-}$. We define $\Delta M \equiv M_{\text {rec }}-m_{D_{s}^{*+}}$, where $m_{D_{s}^{*+}}$ is the nominal $D_{s}^{*+}$ mass [3]. Events within $-0.060<\Delta M<0.065 \mathrm{GeV} / c^{2}$ are accepted as $D_{s}^{*+} D_{s}^{-}$ candidates. To extract the mode-by-mode ST yields, we perform unbinned maximum likelihood fits to the distributions of the $D_{s}^{-}$invariant mass $M_{D_{s}^{-}}$, as shown in Fig. 1. Signals are modeled with the MC-simulated signal shape convoluted with Gaussians to account for the resolution differences between data and MC simulation, while the combinatorial backgrounds are parametrized with secondor third-order polynomial functions. Because of the misidentification of $\pi^{-}$as $K^{-}$, the backgrounds from $D^{-} \rightarrow$ $K_{S}^{0} \pi^{-}$form a broad peak near the $D_{s}^{-}$nominal mass for $D_{s}^{-} \rightarrow K_{S}^{0} K^{-}$. In the fit, the shape of this background is described by using the $\mathrm{MC}$ simulation and its size is set as a free parameter. For each tag mode, the ST yield is obtained by integrating the signal function over the $D_{s}^{-}$mass signal 


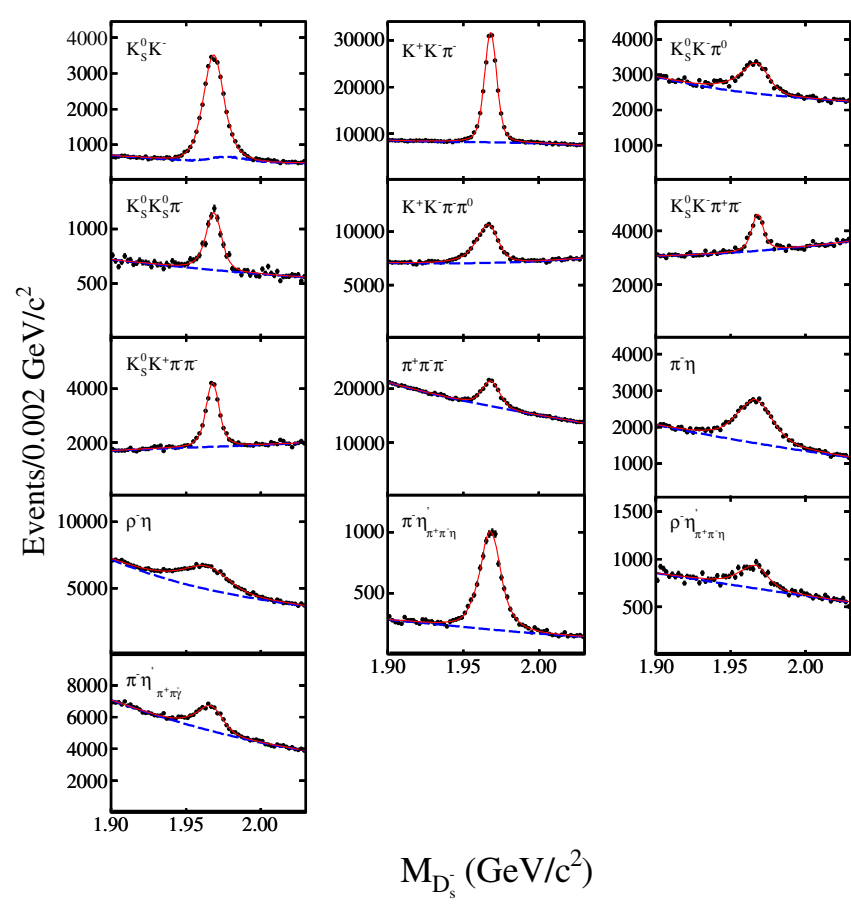

FIG. 1. Fits to $M_{D_{s}^{-}}$distributions for the 13 tag modes. Points with error bars are data, blue dashed curves are the fitted backgrounds, and red solid curves are the total fits.

region specified in the second column of Table I, which also includes the ST yields for all tag modes. The total reconstructed ST yield in our data sample is $N_{\mathrm{ST}}=341$, $325 \pm 1,764$.

In signal events, the system recoiling against the $D_{s}^{-}$tag consists of the SL decay $D_{s}^{+} \rightarrow K^{0} e^{+} \nu_{e}$ or $D_{s}^{+} \rightarrow K^{* 0} e^{+} \nu_{e}$. We select these from the additional tracks accompanying the tag, that is, a $K^{0} \rightarrow K_{S}^{0} \rightarrow \pi^{+} \pi^{-}$with the ST criteria already described, and $K^{* 0} \rightarrow K^{+} \pi^{-}$, therefore, requiring that there be exactly three tracks in the event and with the invariant mass $M_{K^{+} \pi^{-}}$required to be within (0.801, $0.991) \mathrm{GeV} / c^{2}$. Detection and reconstruction of the positron follow the procedures in Refs. $[19,20]$. Backgrounds from $D_{s}^{+} \rightarrow K^{0} \pi^{+}$reconstructed as $D_{s}^{+} \rightarrow K^{0} e^{+} \nu_{e}$ and $D_{s}^{+} \rightarrow K^{+} \pi^{+} \pi^{-}$reconstructed as $D_{s}^{+} \rightarrow K^{* 0} e^{+} \nu_{e}$ are rejected by requiring the $K^{0} e^{+}$or $K^{* 0} e^{+}$invariant mass to be less than $1.78 \mathrm{GeV} / c^{2}$. Backgrounds associated with fake photons are suppressed by requiring $E_{\gamma \max }$, the largest energy of any unused photon, to be less than $0.20 \mathrm{GeV}$.

To identify a photon produced directly from $D_{s}^{* \pm}$, we perform two kinematic fits for each $\gamma$ candidate, one assuming that the $\gamma$ combines with the tag to form a $D_{s}^{*-}$ and the other assuming that the SL decay comes from a $D_{s}^{*+}$ parent. We require the $D_{s}^{\mp} D_{s}^{* \pm}$ pair to conserve energy and momentum in the center-of-mass frame, and the $D_{s}^{ \pm}$ candidates are constrained to the known mass. The neutrino is treated as a missing particle. When we assume the tag to be the daughter of a $D_{s}^{*-}$, we constrain the mass of the photon plus tag candidate to be consistent with the expected $D_{s}^{*-}$ mass; otherwise, we constrain the mass of the photon plus SL decay to be consistent with the $D_{s}^{*+}$ mass. Finally, we select the photon and hypothesis with the smallest kinematic fit $\chi^{2}$.

We obtain information about the undetected neutrino with the missing-mass squared $\left(\mathrm{MM}^{2}\right)$ of the event calculated from the energies and momenta of the tag $\left(E_{D_{s}^{-}}, \vec{p}_{D_{s}^{-}}\right)$, the transition photon $\left(E_{\gamma}, \vec{p}_{\gamma}\right)$, and the detected SL decay products $\left(E_{\mathrm{SL}}=E_{K^{(*) 0}}+E_{e^{+}}, \vec{p}_{\mathrm{SL}}=\vec{p}_{K^{(*) 0}}+\vec{p}_{e^{+}}\right)$ as follows:

$$
\mathrm{MM}^{2}=\left(\sqrt{s}-E_{D_{s}^{-}}-E_{\gamma}-E_{\mathrm{SL}}\right)^{2}-\left(\left|\vec{p}_{D_{s}^{-}}+\vec{p}_{\gamma}+\vec{p}_{\mathrm{SL}}\right|\right)^{2} .
$$

Figure 2 shows the $\mathrm{MM}^{2}$ distributions of the accepted candidate events for $D_{s}^{+} \rightarrow K^{0} e^{+} \nu_{e}$ and $D_{s}^{+} \rightarrow K^{* 0} e^{+} \nu_{e}$ in data. The signal DT yield $N_{\mathrm{DT}}$ is obtained by performing an unbinned maximum likelihood fit to $\mathrm{MM}^{2}$. In the fit, the signal is described with a MC-derived signal shape convolved with a Gaussian, and the background is described by a shape obtained from the inclusive MC sample, in which no peaking backgrounds are observed. We obtain $117.2 \pm 13.9$ and $155.0 \pm 17.2$ events for $D_{s}^{+} \rightarrow K^{0} e^{+} \nu_{e}$ and $D_{s}^{+} \rightarrow K^{* 0} e^{+} \nu_{e}$, respectively, where the uncertainties are statistical only. No peaking backgrounds are observed in $K^{(*) 0}$ mass sideband.

The BFs of $D_{s}^{+} \rightarrow K^{0} e^{+} \nu_{e}$ and $D_{s}^{+} \rightarrow K^{* 0} e^{+} \nu_{e}$ are determined by Eq. (1), where the detection efficiencies $\varepsilon_{\mathrm{SL}}$ are estimated to be $(10.57 \pm 0.04) \%$ and $(19.15 \pm$ $0.06) \%$ for $D_{s}^{+} \rightarrow K^{0} e^{+} \nu_{e}$ and $D_{s}^{+} \rightarrow K^{* 0} e^{+} \nu_{e}$, respectively. (These efficiencies include the BFs for $K^{0} \rightarrow \pi^{+} \pi^{-}$ and $K^{* 0} \rightarrow K^{+} \pi^{-}$.) Finally, we obtain $\mathcal{B}\left(D_{s}^{+} \rightarrow K^{0} e^{+} \nu_{e}\right)=$ $(3.25 \pm 0.38) \times 10^{-3}$ and $\mathcal{B}\left(D_{s}^{+} \rightarrow K^{* 0} e^{+} \nu_{e}\right)=(2.37 \pm$ $0.26) \times 10^{-3}$, where the uncertainties are statistical only.

With the DT technique, the BF measurements are insensitive to the systematic uncertainties of the ST selection. The uncertainties of the $e^{+}$tracking and particle identification (PID) efficiencies have all been determined to be $1.0 \%$ [20], while the uncertainty of the $K^{(*) 0}$ reconstruction is $1.5(2.3) \%$. The uncertainty associated
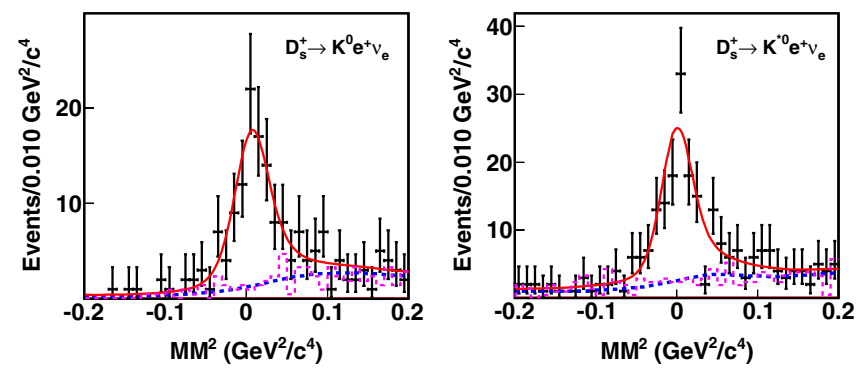

FIG. 2. Fits to $\mathrm{MM}^{2}$ distributions of SL candidate events. Dots with error bars are data, dot-dashed lines (blue) are the fitted backgrounds, and solid curves (red) are the total fits. The longdashed lines (pink) show the backgrounds from the $M_{D_{s}^{-}}$sidebands. 
TABLE II. FF results from fits to $D_{s}^{+} \rightarrow K^{0} e^{+} \nu_{e}$, where the first errors are statistical and the second systematic.

\begin{tabular}{lcc}
\hline \hline Parametrizations & $f_{+}^{K}(0)\left|V_{c d}\right|$ & $f_{+}^{K}(0)$ \\
\hline Simple pole [25] & $0.172 \pm 0.010 \pm 0.001$ & $0.765 \pm 0.044 \pm 0.004$ \\
Modified pole [25] & $0.163 \pm 0.017 \pm 0.003$ & $0.725 \pm 0.076 \pm 0.013$ \\
$z$ series (two-parameter) [26] & $0.162 \pm 0.019 \pm 0.003$ & $0.720 \pm 0.084 \pm 0.013$ \\
\hline \hline
\end{tabular}

with the $\mathrm{MM}^{2}$ fit is estimated to be 3.5 (3.8)\% by varying the fitting ranges and the signal and background shapes. The uncertainty due to the selection of the $\gamma$ is estimated to be $2.0 \%$ based on selecting the best photon candidate in a control sample of $e^{+} e^{-} \rightarrow D_{s}^{+*} D_{s}^{-}$events with two hadronic tags, $D_{s}^{+} \rightarrow K_{S}^{0} K^{+}$and $D_{s}^{-} \rightarrow K^{+} K^{-} \pi^{-}$. The uncertainties due to the $E_{\gamma \max }$ and $M_{K^{(*)} e^{+}}$requirements are estimated to be $1.7(1.7) \%$ and $0.7(0.9) \%$ by comparing the nominal $\mathrm{BF}$ with that measured with alternative requirements. The uncertainty due to the MC signal modeling is estimated to be 0.9 (1.8)\% by varying the input FF parameters by $\pm 1 \sigma$ as determined in this work. We also consider the systematic uncertainties of $N_{\mathrm{ST}}(0.5 \%)$ evaluated by using alternative signal shapes when fitting the $M_{D_{s}^{-}}$ spectra and of the MC statistics $(0.4 \%)$. The uncertainty due to different tag dependences between data and MC simulation is estimated to be $0.8(0.3) \%$. Additionally, for $D_{s}^{+} \rightarrow K^{* 0} e^{+} \nu_{e}$ decay, the systematic uncertainty for the possible $\mathcal{S}$-wave component in the $K \pi$ system is estimated to be $6.0 \%$ according to Refs. [21,22]. Adding these contributions in quadrature gives total systematic uncertainties of $5.1 \%$ and $8.3 \%$ for $\mathcal{B}\left(D_{s}^{+} \rightarrow K^{0} e^{+} \nu_{e}\right)$ and $\mathcal{B}\left(D_{s}^{+} \rightarrow K^{* 0} e^{+} \nu_{e}\right)$, respectively.

The $D_{s}^{+} \rightarrow K^{0} e^{+} \nu_{e}$ differential decay width with respect to the mass squared $\left(q^{2}\right)$ of the $e^{+} \nu_{e}$ system is expressed as [23]

$$
\frac{d \Gamma\left(D_{s}^{+} \rightarrow K^{0} e^{+} \nu_{e}\right)}{d q^{2}}=\frac{G_{F}^{2}\left|V_{c d}\right|^{2}}{24 \pi^{3}} p_{K^{0}}^{3}\left|f_{+}^{K}\left(q^{2}\right)\right|^{2} .
$$

In this equation, $p_{K^{0}}$ is the $K^{0}$ momentum in the rest frame of the $D_{s}^{+}, G_{F}$ is the Fermi constant [3], $\left|V_{c d}\right|$ is the CKM matrix element, and $f_{+}^{K}\left(q^{2}\right)$ is the hadronic FF. To extract the FF parameters, we fit to the differential decay rates $\Delta \Gamma_{i}$ measured in the $q^{2}$ bins of $[0.00,0.35),[0.35,0.70),[0.70$, $1.05),[1.05,1.40)$, and $[1.40,2.16) \mathrm{GeV} / c^{2}$ by using the three theoretical parametrizations in Table II. A least- $\chi^{2}$ fit is performed accounting for correlations among $q^{2}$ bins. We fix the pole mass $m_{\text {pole }}$ at the $D^{*+}$ nominal mass [3]. The fits to the differential decay rate and projections of the fits onto $f_{+}\left(q^{2}\right)$ for $D_{s}^{+} \rightarrow K^{0} e^{+} \nu_{e}$ are shown in Figs. 3(a) and $3(\mathrm{~b})$, and the FF fit results are summarized in the third column of Table II. The systematic uncertainties in the extracted parameters are estimated as in Ref. [24]. These include the same systematic effects as the BF measurements, along with the $D_{s}^{+}$-lifetime uncertainty. Using $\left|V_{c d}\right|=0.22492 \pm 0.00050$ [3], we obtain $f_{+}^{K}(0)$ as shown in the last column of Table II.

The differential decay rate of $D_{s}^{+} \rightarrow K^{* 0} e^{+} \nu_{e}$ depends on five variables: $K \pi$ mass squared $\left(m_{K \pi}^{2}\right), e^{+} \nu_{e}$ mass squared $\left(q^{2}\right)$, the angle between the $K^{+}$and $D_{s}^{+}$momenta in the $K \pi$ rest frame $\left(\theta_{K}\right)$, the angle between the $\nu_{e}$ and $D_{s}^{+}$ momenta in the $e^{+} \nu_{e}$ system $\left(\theta_{e}\right)$, and the acoplanarity angle between the $K \pi$ and $e^{+} \nu_{e}$ decay planes $(\chi)$. The differential decay rate can be expressed in terms of three helicity amplitudes [27,28]: $H_{ \pm}\left(q^{2}\right)=\left(M_{D_{s}^{+}}+\right.$ $\left.m_{K \pi}\right) A_{1}\left(q^{2}\right) \mp\left[\left(2 M_{D_{s}^{+}} P_{K \pi}\right) /\left(M_{D_{s}^{+}}+M_{K \pi}\right)\right] V\left(q^{2}\right)$ and $H_{0}\left(q^{2}\right)=\left(1 / 2 m_{K \pi} q\right)\left[\left(M_{D_{s}^{+}}^{2}-m_{K \pi}^{2}-q^{2}\right)\left(M_{D_{s}^{+}}+m_{K \pi}\right) A_{1}\left(q^{2}\right)-\right.$ $\left.\left[\left(4 M_{D_{s}^{+}}^{2} p_{K \pi}^{2}\right) /\left(M_{D_{s}^{+}}+M_{K \pi}\right)\right] A_{2}\left(q^{2}\right)\right]$, where $p_{K \pi}$ is the
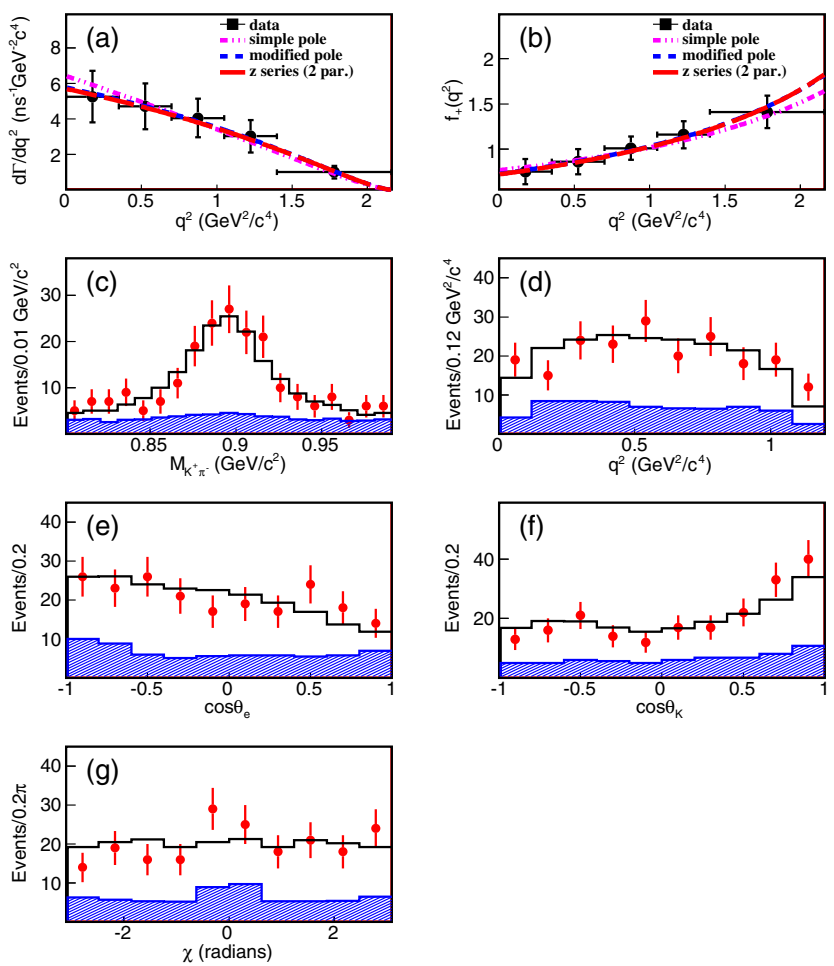

FIG. 3. (a) Fits to the differential decay rates and (b) projections onto $f_{+}^{K}\left(q^{2}\right)$ for $D_{s}^{+} \rightarrow K^{0} e^{+} \nu_{e}$. Projections onto (c) $M_{K^{+} \pi^{-}}$, (d) $q^{2}$, (e) $\cos \theta_{e}$, (f) $\cos \theta_{K}$, and (g) $\chi$ for $D_{s}^{+} \rightarrow K^{* 0} e^{+} \nu_{e}$. Dots with error bars are data. Curves in (a),(b) give the best fits with different FF parametrizations. Solid and shadowed histograms in (c) $-(\mathrm{g})$ are the $\mathrm{MC}$-simulated signal plus background and the MC-simulated background. 
momentum of the $K \pi$ system in the rest frame of the $D_{s}^{+}$, and $V\left(q^{2}\right)$ and $A_{1 / 2}\left(q^{2}\right)$ are the vector and axial FFs, respectively. Because $A_{1}\left(q^{2}\right)$ is common to all three helicity amplitudes, it is natural to define the FF ratios $r_{V}=$ $V(0) / A_{1}(0)$ and $r_{2}=A_{2}(0) / A_{1}(0)$. The $A_{1 / 2}\left(q^{2}\right)$ and $V\left(q^{2}\right)$ are assumed to have simple pole forms, $A_{1 / 2}\left(q^{2}\right)=$ $A_{1 / 2}(0) /\left(1-q^{2} / M_{A}^{2}\right)$ and $V\left(q^{2}\right)=V(0) /\left(1-q^{2} / M_{V}^{2}\right)$, with pole masses $M_{V}=M_{D^{*}\left(1^{-}\right)}=2.01 \mathrm{GeV} / c^{2}$ and $M_{A}=M_{D^{*}\left(1^{+}\right)}=2.42 \mathrm{GeV} / c^{2}[3]$.

We perform a five-dimensional maximum likelihood fit in the space of $M_{K^{+} \pi^{-}}^{2}, q^{2}, \cos \theta_{e}, \cos \theta_{K}$, and $\chi$ for the $D_{s}^{+} \rightarrow K^{* 0} e^{+} \nu_{e}$ events within $-0.15<\mathrm{MM}^{2}<0.15 \mathrm{GeV}^{2} / c^{4}$ in a similar manner as Refs. [27,28]. We ignored the possible $\mathcal{S}$-wave component in the $K \pi$ system due to limited statistics. The projections of the fit onto $M_{K^{+} \pi^{-}}^{2}$, $q^{2}, \cos \theta_{e}, \cos \theta_{K}$, and $\chi$ are shown in Figs. 3(c)-3(g). In this fit, the $K^{* 0}$ Breit-Wigner function follows Ref. [27], with a mass and width fixed to those reported in Ref. [3]. We obtain $r_{V}=1.67 \pm 0.34$ (stat) and $r_{2}=$ $0.77 \pm 0.28$ (stat). The fit procedure has been validated by analyzing a large inclusive MC sample, and the pull distribution of each fitted parameter was consistent with a normal distribution. The systematic uncertainties in the FF ratio measurements are estimated by comparing the nominal values with those obtained after varying one source of uncertainty, as described in Ref. [22]. The systematic uncertainties in measuring $r_{V}\left(r_{2}\right)$ arise mainly from the uncertainties related to tracking, PID, and photon detection (1.8\%), the $K^{* 0}$ mass window (1.8\%), the $\mathrm{MM}^{2}$ signal region $(8.7 \%)$, the $E_{\gamma \max }$ requirement $(1.2 \%)$, the $M_{K^{* 0} e^{+}}$requirement (0.6\%), background estimation (1.8\%), and the $K^{* 0}$ Breit-Wigner line shape $(0.3 \%)$. Combining all of these in quadrature, we find the systematic uncertainties in $r_{V}$ and $r_{2}$ of $D_{s}^{+} \rightarrow K^{* 0} e^{+} \nu_{e}$ to be $9.3 \%$ and $8.7 \%$, respectively.

In summary, using $3.19 \mathrm{fb}^{-1}$ data collected at $\sqrt{s}=$ $4.178 \mathrm{GeV}$ by the BESIII detector, we measure the absolute BFs of $D_{s}^{+} \rightarrow K^{0} e^{+} \nu_{e}$ and $D_{s}^{+} \rightarrow K^{* 0} e^{+} \nu_{e}$ to be $\mathcal{B}\left(D_{s}^{+} \rightarrow K^{0} e^{+} \nu_{e}\right)=[3.25 \pm 0.38$ (stat) \pm 0.16 (syst) $] \times 10^{-3}$ and $\mathcal{B}\left(D_{s}^{+} \rightarrow K^{* 0} e^{+} \nu_{e}\right)=[2.37 \pm 0.26$ (stat) \pm 0.20 (syst) $] \times$ $10^{-3}$. These are the most precise measurements to date. Theoretical predictions of these BFs range from $2.0 \times 10^{-3}$ to $3.9 \times 10^{-3}[23,29-33]$ for $D_{s}^{+} \rightarrow K^{0} e^{+} \nu_{e}$ and $1.7 \times 10^{-3}$ to $2.3 \times 10^{-3}[23,30-34]$ for $D_{s}^{+} \rightarrow K^{* 0} e^{+} \nu_{e}$, respectively. Since the predicated BF $2.0 \times 10^{-3}$ in Refs. [29,33] obtained from a double-pole model are more than 2 standard deviations away from the mean value of our measured $\mathcal{B}\left(D_{s}^{+} \rightarrow K^{0} e^{+} \nu_{e}\right)$, thus, at a confidence level of $95 \%$, our measurement disfavors this prediction.

By analyzing the dynamics of $D_{s}^{+} \rightarrow K^{0} e^{+} \nu_{e}$ and $D_{s}^{+} \rightarrow$ $K^{* 0} e^{+} \nu_{e}$ decays for the first time, we determine the FF of $D_{s}^{+} \rightarrow K^{0} e^{+} \nu_{e} \quad$ to be $f_{+}^{K}(0)=0.720 \pm 0.084$ (stat) \pm 0.013 (syst) and the FF ratios of $D_{s}^{+} \rightarrow K^{* 0} e^{+} \nu_{e}$ to be
TABLE III. The ratios of the from factors.

\begin{tabular}{lc}
\hline \hline & Values \\
\hline$f_{+}^{D_{s}^{+} \rightarrow K^{0}}(0) / f_{+}^{D^{+} \rightarrow \pi^{0}}(0)$ & $1.16 \pm 0.14($ stat $) \pm 0.02$ (syst) \\
$r_{V}^{D_{s}^{+} \rightarrow K^{* 0}} / r_{V}^{D^{+} \rightarrow \rho^{0}}$ & $1.13 \pm 0.26($ stat $) \pm 0.11$ (syst) \\
$r_{2}^{D_{s}^{+} \rightarrow K^{* 0}} / r_{2}^{D^{+} \rightarrow \rho^{0}}$ & $0.93 \pm 0.36($ stat $) \pm 0.10$ (syst) \\
\hline \hline
\end{tabular}

$r_{V}=1.67 \pm 0.34$ (stat) \pm 0.16 (syst) $\quad$ and $\quad r_{2}=0.77 \pm$ 0.28 (stat) \pm 0.07 (syst). With the FF of $D^{+} \rightarrow \pi^{0} e^{+} \nu_{e}$ measured by BESIII [24] and that of $D^{+} \rightarrow \rho^{0} e^{+} \nu_{e}$ by CLEO [27], we calculate the ratios of the FFs of $D_{s}^{+} \rightarrow$ $K^{0} e^{+} \nu_{e}$ to $D^{+} \rightarrow \pi^{0} e^{+} \nu_{e}$ and $D_{s}^{+} \rightarrow K^{* 0} e^{+} \nu_{e}$ to $D^{+} \rightarrow$ $\rho^{0} e^{+} \nu_{e}$ decays, as shown in Table III, which are consistent with LQCD predictions [4] and the expectation of $U$-spin $(d \leftrightarrow s)$ symmetry [35]. These measurements provide a first test of the LQCD prediction that the FFs are insensitive to spectator quarks, which has important implications when considering the corresponding $B$ and $B_{s}$ decays [4-6].

The BESIII Collaboration thanks the staff of BEPCII and the IHEP computing center for their strong support. This work is supported in part by National Key Basic Research Program of China under Contract No. 2015CB856700; National Natural Science Foundation of China (NSFC) under Contracts No. 11335008, No. 11425524, No. 11505010, No. 11625523, No. 11635010, No. 11735014, and No. 11775027; the Chinese Academy of Sciences (CAS) Large-Scale Scientific Facility Program; the CAS Center for Excellence in Particle Physics; Joint Large-Scale Scientific Facility Funds of the NSFC and CAS under Contracts No. U1532257, No. U1532258, and No. U1732263; CAS Key Research Program of Frontier Sciences under Contracts No. QYZDJ-SSW-SLH003 and No. QYZDJ-SSW-SLH040; 100 Talents Program of CAS; Tsung-Dao Lee Institute and Shanghai Key Laboratory for Particle Physics and Cosmology; German Research Foundation DFG under Contracts No. Collaborative Research Center CRC 1044 and No. FOR 2359; Istituto Nazionale di Fisica Nucleare, Italy; Koninklijke Nederlandse Akademie van Wetenschappen under Contract No. 530-4CDP03; Ministry of Development of Turkey under Contract No. DPT2006K-120470; National Science and Technology fund; The Swedish Research Council; U.S. Department of Energy under Contracts No. DE-FG0205ER41374, No. DE-SC-0010118, No. DE-SC-0010504, and No. DE-SC-0012069; University of Groningen and the Helmholtzzentrum fuer Schwerionenforschung $\mathrm{GmbH}$, Darmstadt. This paper is also supported by the Beijing municipal government under Contracts No. KM201610017009, No. 2015000020124G064, and No. CIT\&TCD201704047, and by the Royal Society under the Newton International Fellowship Contract No. NF170002. 
*lilei2014@bipt.edu.cn

aAlso at Bogazici University, 34342 Istanbul, Turkey.

${ }^{\mathrm{b}}$ Also at the Moscow Institute of Physics and Technology, Moscow 141700, Russia.

${ }^{\mathrm{c}}$ Also at the Functional Electronics Laboratory, Tomsk State University, Tomsk, 634050, Russia.

${ }^{\mathrm{d}}$ Also at the Novosibirsk State University, Novosibirsk, 630090, Russia.

"Also at the NRC "Kurchatov Institute", PNPI, 188300, Gatchina, Russia.

${ }^{\mathrm{f}}$ Also at Istanbul Arel University, 34295 Istanbul, Turkey.

${ }^{\mathrm{g}}$ Also at Goethe University Frankfurt, 60323 Frankfurt am Main, Germany.

${ }^{\mathrm{h}}$ Also at Key Laboratory for Particle Physics, Astrophysics and Cosmology, Ministry of Education; Shanghai Key Laboratory for Particle Physics and Cosmology; Institute of Nuclear and Particle Physics, Shanghai 200240, People's Republic of China.

${ }^{\mathrm{i}}$ Also at Government College Women University, Sialkot51310, Punjab, Pakistan.

${ }^{\mathrm{j} A l s o}$ at Key Laboratory of Nuclear Physics and Ion-beam Application (MOE) and Institute of Modern Physics, Fudan University, Shanghai 200443, People's Republic of China.

[1] M. B. Voloshin, Phys. Lett. B 515, 74 (2001); D. Scora and N. Isgur, Phys. Rev. D 52, 2783 (1995).

[2] J. Yelton et al. (CLEO Collaboration), Phys. Rev. D 80, 052007 (2009).

[3] C. Patrignani et al. (Particle Data Group), Chin. Phys. C 40, 100001 (2016) and 2017 update.

[4] J. Koponen, C. T. H. Davies, and G. Donald (HPQCD Collaboration), arXiv:1208.6242; J. Koponen, C. T. H. Davies, G. C. Donald, E. Follana, G. P. Lepage, H. Na, and J. Shigemitsu (HPQCD Collaboration), arXiv:1305.1462.

[5] N. Brambilla et al., Eur. Phys. J. C 74, 2981 (2014).

[6] J. A. Bailey, A. Bazavov, C. Bernard et al., Phys. Rev. D 85, 114502 (2012).

[7] M. Ablikim et al. (BESIII Collaboration), Nucl. Instrum. Methods Phys. Res., Sect. A 614, 345 (2010).

[8] S. Agostinelli et al. (GEANT4 Collaboration), Nucl. Instrum. Methods Phys. Res., Sect. A 506, 250 (2003).

[9] R. G. Ping, Chin. Phys. C 38, 083001 (2014).

[10] D. J. Lange, Nucl. Instrum. Methods Phys. Res., Sect. A 462, 152 (2001); 7R. G. Ping, Chin. Phys. C 32, 599 (2008).

[11] E. A. Kurav and V. S. Fadin, Yad. Fiz. 41, 733 (1985) [Sov. J. Nucl. Phys. 41, 466 (1985)].
[12] E. Richter-Was, Phys. Lett. B 303, 163 (1993).

[13] S. Jadach, B. F. L. Ward, and Z. Was, Phys. Rev. D 63, 113009 (2001); D. Bardin, M. Bilenky, A. Chizhov, A. Sazonov, O. Fedorenko, T. Riemann, and M. Sachwitz, Nucl. Phys. B351, 1 (1991).

[14] K. A. Olive et al. (Particle Data Group), Chin. Phys. C 38, 090001 (2014) and 2015 update.

[15] J. C. Chen, G. S. Huang, X. R. Qi, D. H. Zhang, and Y. S. Zhu, Phys. Rev. D 62, 034003 (2000).

[16] D. Cronin-Hennessy et al. (CLEO Collaboration), Phys. Rev. D 80, 072001 (2009).

[17] M. Ablikim et al. (BESIII Collaboration), Phys. Rev. Lett. 118, 112001 (2017); Phys. Lett. B 772, 388 (2017).

[18] M. Ablikim et al. (BESIII Collaboration), Phys. Rev. D 99, 011103(R) (2019).

[19] M. Ablikim et al. (BESIII Collaboration), Phys. Rev. D 92, 112008 (2015); 92, 071101(R) (2015).

[20] M. Ablikim et al. (BESIII Collaboration), Phys. Rev. Lett. 115, 221805 (2015).

[21] P. del Amo Sanchez et al. (BABAR Collaboration), Phys. Rev. D 83, 072001 (2011).

[22] M. Ablikim et al. (BESIII Collaboration), Phys. Rev. D 94, 032001 (2016).

[23] W. Wang and Y.L. Shen, Phys. Rev. D 78, 054002 (2008).

[24] M. Ablikim et al. (BESIII Collaboration), Phys. Rev. D 96, 012002 (2017).

[25] D. Becirevcic and A. B. Kaidalov, Phys. Lett. B 478, 417 (2000).

[26] T. Becher and R. J. Hill, Phys. Lett. B 633, 61 (2006).

[27] S. Dobbs et al. (CLEO Collaboration), Phys. Rev. Lett. 110, 131802 (2013).

[28] M. Ablikim et al. (BESIII Collaboration), Phys. Rev. D 92 , 071101(R) (2015).

[29] S. Fajfer and J. Kamenik, Phys. Rev. D 71, 014020 (2005).

[30] D. Melikhov and B. Stech, Phys. Rev. D 62, 014006 (2000).

[31] Y. L. Wu, M. Zhong, and Y. B. Zuo, Int. J. Mod. Phys. A 21, 6125 (2006).

[32] H. Y. Cheng and X. W. Kang, Eur. Phys. J. C 77, 587 (2017).

[33] N. R. Soni, M. A. Ivanov, J. G. Körner, J. N. Pandya, P. Santorelli, and C. T. Tran, Phys. Rev. D 98, 114031 (2018).

[34] S. Fajfer and J. Kamenik, Phys. Rev. D 72, 034029 (2005).

[35] M. Gronau, Phys. Lett. B 492, 297 (2000). 